ARTÍCULO DE INVESTIGACIÓN

\title{
Componentes químicos de la madera de cinco especies de pino del municipio de Morelia, Michoacán
}

\section{Chemical components of the wood of five pine species of Morelia, Michoacán}

\author{
René Bernabé-Santiago ${ }^{1}$, Luz Elena A. Ávila-Calderón ${ }^{2}$ \\ y José Guadalupe Rutiaga-Quiñones²
}

\begin{abstract}
RESUMEN
Se realizó un análisis para determinar los principales componentes químicos de la madera de cinco especies de pino procedentes del municipio de Morelia, Michoacán. Las especies estudiadas fueron Pinus leiophylla, $P$. michoacana var. cornuta, $P$. montezumae, $P$. oocarpa y $P$. teocote. En este estudio se determinó en la madera de cada especie el $\mathrm{pH}$, las cenizas, el análisis de las cenizas, las sustancias extraíbles, la holocelulosa, la lignina y los taninos. Los resultados encontrados fueron: $\mathrm{pH}$ de 4,0 a 4,$4 ; 0,3 \%$ de cenizas, con mayor concentración de calcio, potasio y magnesio, $17,9 \%$ a $25,4 \%$ de solubilidad en sosa; $7,6 \%$ a $8,2 \%$ de extraíbles totales, en la extracción secuencial, la mayor solubilidad se obtuvo con agua caliente, seguido en proporciones similares con ciclohexano, acetona y metanol; $68,1 \%$ a $74,7 \%$ de holocelulosa, $24,0 \%$ a $28,5 \%$ de lignina y $0,07 \%$ a $0,12 \%$ para taninos. La solubilidad de la madera para las extracciones siguió la secuencia agua > ciclohexano $>$ acetona $>$ metanol. El pH, la solubilidad a la sosa, la holocelulosa y la lignina presentaron una variación estadística significativa $(P<0,05)$ entre especies.
\end{abstract}

PALABRAS CLAVE:

Extraíbles, holocelulosa, lignina, Pinus spp., sustancias inorgánicas, taninos.

\begin{abstract}
A chemical analysis was performed to determine the major chemical components of five pine species in the municipality of Morelia, Michoacán. The pine species studied were: Pinus leiophylla, $P$. michoacana var. cornuta, $P$. montezumae, $P$. oocarpa and $P$. teocote. The $\mathrm{pH}$ value, ash, ash analysis, extractives, holocellulose, lignin and tannins content was determined for each pine species. The results were in the range of 4,0 to 4,4 for $\mathrm{pH}, 0,3 \%$ ash in all species (calcium and potassium in higher concentration), $17,9 \%$ to $25,4 \%$ for soda solubility, $7,6 \%$ to $8,2 \%$ total extractives, $68,1 \%$ to $74,7 \%$ for holocellulose, $24 \%$ to $28,5 \%$ for lignin and $0,07 \%$ to $0,12 \%$ for tannins. Through the applied extraction the wood solubility followed the sequence: water $>$ ciclohexan $>$ aceton $>$ methanol. Analysis of variance of the data showed that $\mathrm{pH}$, soda solubility, holocellulose, lignin and tannins did vary among pine species $(P<0,05)$.
\end{abstract}

KEY WORDS:

Extractives, holocellulose, lignin, Pinus spp., inorganic substances, tannins. Hidalgo.

2 Facultad de Ingeniería en Tecnología de la Madera. Universidad Michoacana de San Nicolás de Hidalgo. Apdo. Postal 580. C. P. 58000 Morelia, Michoacán, México. 


\section{INTRODUCCIÓN}

Los pinos representan uno de los recursos naturales más valiosos para México; la madera de escuadría es el producto más importante (3 337940 metros cúbicos rollo), seguido de productos celulósicos (346 510 metros cúbicos rollo), postes, pilotes y durmientes (187 797 metros cúbicos rollo) y chapa y triplay (183 571 metros cúbicos rollo) (Semarnat, 2012). En México existen 46 especies, 3 subespecies y 22 variedades de pinos, que representan $42 \%$ de las especies de este género conocidas en el mundo (Sánchez, 2008). De acuerdo con Madrigal y Guridi (2002), se conocen 20 especies del género Pinus en el estado de Michoacán, de las cuales 11 se encuentran en el municipio de Morelia. Algunos estudios referentes a este género han abarcado temas como: características anatómicas (Olvera 1981, 1985; Ambriz et al., 2002; Ochoa, 2003), propiedades físico-mecánicas (Herrera y Bocanegra, 1996; Ortega, 2001; Rojas y Villers, 2005; Silva-Arredondo y Návar-Cháidez, 2012) y propiedades tecnológicas (Cruz y Ambriz, 2004; Aquino et al., 2010).

La composición química de la madera de los pinos mexicanos se ha estudiado para pocas especies. En 2001 Rutiaga estimó en madera de albura y duramen de $P$. pseudostrobus la solubilidad en éter de petróleo $(0,7 \%$ y $4,4 \%)$, acetona $(0,7 \%$ y $2,8 \%)$, metanol $(0,8 \%$ y $0,8 \%$, agua fría $(1,0 \%$ y $3,7 \%)$ y agua caliente $(1,2 \%$ y $1,5 \%)$; el contenido de polisacáridos $(67,8 \%$ y $67,2 \%)$, de lignina Runkel $(26,6 \%$ y $27,6 \%)$ y de cenizas $(0,16 \%$ y $0,08 \%)$, además de identificar los elementos presentes en estas últimas.

Ávila (2011) estudió los componentes químicos de la madera de $P$. pringlei sana e infectada por muérdago Psittacanthus macrantherus. Encontró que los contenidos de sustancias inorgánicas
$(0,34 \%)$, de extraíbles solubles en etanolciclohexano $(0,83 \%)$, de extraíbles solubles en agua fría $(3,62 \%)$ y de holocelulosa $(63,58 \%)$ resultaron ser mayores en madera sana que en madera plagada $(0,31 \%, 0,67 \%, 3,09 \%$ y $42,82 \%$ respectivamente); por el contrario, la madera infectada presentó valores más altos de solubilidad en sosa $(9,87 \%)$, de extraíbles solubles en agua caliente $(6,22 \%)$ y de lignina $(28,50 \%)$ en relación con la madera sana $(8,46 \%, 5,29 \%$ y $23,56 \%$ respectivamente).

Ravilla (2011) estudió algunos aspectos de la química de la madera de $P$. cembroides, $P$. johannis, $P$. maximartinezii y $P$. pinceana, cuantificando $\mathrm{pH}(4,09-$ $5,63)$, cenizas $(<0,6 \%)$ de las que $90 \%$ fueron potasio, calcio y magnesio, contenidos de extraíbles altos especialmente en el duramen; bajo contenido de celulosa (35,5\% - 37,9\% determinado por hidrólisis de carbohidratos y $34,8 \%-37,1 \%$ determinado por deslignificación), alto contenido de lignina $(28,8 \%-31,5 \%$ y $32,6 \%$ - $36,4 \%)$ y alto contenido de hemicelulosas $(29,4 \%-32,2 \%$ y $28,8 \%-30,3 \%)$, determinó además las hemicelulosas presentes (glucomanana 15,6\% - 20,6\% y glucuronoxilana $7,2 \%-10,2 \%$ ).

Dada la poca información que se tiene de los componentes químicos de las maderas mexicanas de pino, son necesarios estudios que, además de aportar elementos para su mejor aprovechamiento, permitan establecer interrelaciones con propiedades físicas, mecánicas y tecnológicas de este valioso recurso.

\section{OBJETIVO}

Determinar los componentes químicos básicos de la madera de Pinus leiophylla Schl. et Cham, P. michoacana var. cornuta Martínez, $P$. montezumae Lamb., $P$. oocarpa Schiede y $P$. teocote Schl. et 
Cham., procedentes del municipio de Morelia, Michoacán, con el propósito de contribuir al conocimiento químico de los pinos del país.

\section{METODOLOGÍA}

\section{Colecta y habilitación del material}

La información sobre las especies en estudio, localidad de colecta, respaldo de herbario (Herbario de la Facultad de Biología de la Universidad Michoacana EBUM) y de xiloteca (colección de tablillas de xiloteca de la Facultad de Ingeniería en Tecnología de la Madera) se muestran en la Tabla 1.

De un ejemplar de cada especie en estudio se obtuvo una rodaja de $20 \mathrm{~cm}$ de espesor a una altura de 1,30 m. Del material (mezcla de albura y duramen) se obtuvieron tablillas de $1 \mathrm{~cm} \times 6 \mathrm{~cm} \times 10 \mathrm{~cm}$, que fueron astilladas, secadas al aire y molidas en un equipo Wiley. La harina obtenida se clasificó con tamices, para el análisis químico se empleó la fracción que pasó por la malla $40(425 \mu \mathrm{m})$ y que fue retenida en la malla $60(250 \mu \mathrm{m})$. Las determinaciones se realizaron por duplicado.

\section{Análisis químico}

$p H$. La determinación del pH (Moisture $\mathrm{pH} ; \mathrm{MpH}$ ) se basó en el método de Sandermann y Rothkamm (1959); 2 g de harina de madera sin extraer se colocaron en un vaso de precipitados con $20 \mathrm{ml}$ de agua destilada, se registró la lectura inicial del $\mathrm{pH}$ con un potenciómetro marca HANNA y se volvió a tomar a los 5 minutos, a las 4 horas, a las 24 horas y a las 48 horas de comenzada la medición.

Sustancias inorgánicas. El contenido de estas sustancias se calculó gravimétricamente, después de quemar cuidadosa- mente $2 \mathrm{~g}$ de harina de madera sin extraer en un crisol de níquel, previamente tarado, sobre una placa de calentamiento hasta el cese de desprendimiento de humo, para su posterior calcinación en una mufla a $525^{\circ} \mathrm{C}$; el proceso se dio por concluido hasta que el crisol tuvo peso constante, el procedimiento y cálculo de las sustancias inorgánicas o cenizas se realizó de acuerdo con la norma T 211 om-93 (TAPPI, 2000a).

Para la identificación de los elementos presentes en las sustancias inorgánicas se realizó su microanálisis en un espectrómetro de Rayos X, acoplado a un microscopio electrónico de barrido marca Jeol modelo JSM- 6400. Las condiciones de operación para los análisis fueron 20 kV y 8,5 segundos, obteniéndose los espectros respectivos en puntos aleatorios eliminándose el carbono y oxígeno que pudieran haber quedado como residuo de los componentes orgánicos.

Solubilidad en sosa. En un matraz se colocaron $2 \mathrm{~g}$ de harina de madera y $100 \mathrm{ml}$ de $\mathrm{NaOH}$ al 1,0\%; se llevaron a digestión en baño de agua durante 60 minutos. Se agitó la harina con una varilla de vidrio durante aproximadamente $5 \mathrm{~s}$ en $10 \mathrm{~min}$, 15 min y 25 min después de la colocación en el baño. Al final de la digestión, el material se filtró y lavó con $100 \mathrm{ml}$ de agua caliente, posteriormente se neutralizó en dos etapas con $25 \mathrm{ml}$ de ácido acético al $10 \%$ y se lavó nuevamente con agua caliente hasta dejar el material libre de ácido. Finalmente, se secó el filtro y su contenido a $105{ }^{\circ} \mathrm{C}$ hasta obtener peso constante. El proceso y el cálculo de la solubilidad en sosa se efectuó siguiendo la norma T 212 om-98 (TAPPI, 2000b).

Sustancias extraíbles. Para determinar la cantidad total de sustancias extraíbles $(T)$, en $6 \mathrm{~g}$ de harina de madera se aplicó una extracción sucesiva sólido-líquido en equipo Soxhlet con $200 \mathrm{ml}$ de los siguientes 


\begin{tabular}{|c|c|c|c|c|c|}
\hline 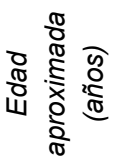 & $\hat{10}$ & $\infty$ & $\stackrel{2}{\sim}$ & 음 & $\stackrel{10}{R}$ \\
\hline ¿ & ষे & ஜి & ిొ & \& & ి్ల \\
\hline 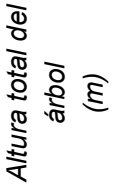 & $\stackrel{\bullet}{\circ}$ & ㅇ & $\stackrel{\sim}{\sim}$ & $\stackrel{\nabla}{\sim}$ & $\stackrel{\infty}{\underset{\sim}{\sim}}$ \\
\hline 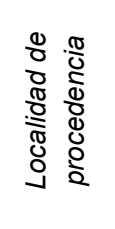 & 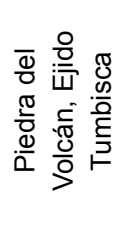 & 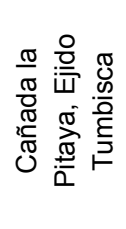 & 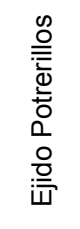 & $\frac{\stackrel{D}{N}}{\stackrel{N}{E}}$ & 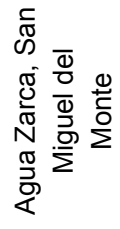 \\
\hline 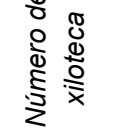 & $\stackrel{R}{\stackrel{R}{\vdash}}$ & $\stackrel{\hat{\imath}}{\vdash}$ & $\begin{array}{l}\infty \\
\infty \\
\stackrel{1}{1} \\
⺊\end{array}$ & $\frac{\stackrel{N}{\circ}}{\vdash}$ & $\stackrel{\stackrel{p}{P}}{\stackrel{P}{\vdash}}$ \\
\hline 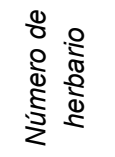 & $\begin{array}{l}\text { J } \\
\stackrel{+}{+} \\
1 \\
I\end{array}$ & \begin{tabular}{l}
\multirow{O}{O}{} \\
$\frac{0}{+}$ \\
1 \\
1
\end{tabular} & $\begin{array}{l}\stackrel{\text { N }}{+} \\
1 \\
I\end{array}$ & $\begin{array}{l}0 \\
\stackrel{+}{+} \\
1 \\
I\end{array}$ & $\begin{array}{l}\text { \$ } \\
\text { D } \\
1 \\
1 \\
1\end{array}$ \\
\hline 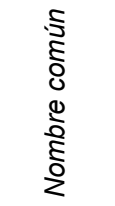 & 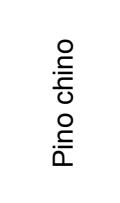 & $\begin{array}{l}\frac{0}{0} \\
\frac{\pi}{0} \\
0 \\
\stackrel{0}{0}\end{array}$ & $\begin{array}{l}\frac{0}{0} \\
\frac{\pi}{0} \\
\stackrel{0}{=} \\
\frac{.0}{2}\end{array}$ & 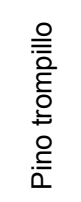 & 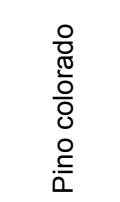 \\
\hline 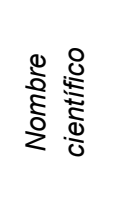 & 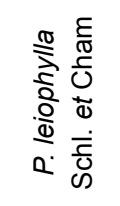 & 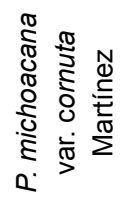 & 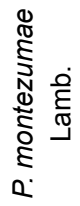 & 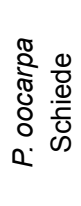 & 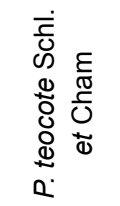 \\
\hline
\end{tabular}


solventes: ciclohexano (CH), acetona (ACE) y metanol (MET), finalmente, agua caliente bajo reflujo (AC). Los periodos de extracción fueron de cuatro horas. Los solventes se recuperaron en un rotavapor aplicando vacío y el extracto respectivo se colocó en un desecador, con gel de sílice como agente desecante, hasta peso constante. El contenido de extraíbles para cada solvente se calculó dividiendo el peso del extracto anhidro entre el peso de la harina anhidra referido porcentualmente. Los extraíbles totales se calcularon con la suma de los porcentajes de los extraíbles de cada solvente. La harina de madera, después de la extracción sucesiva, se designó como harina libre de extraíbles y se empleó para determinar lignina y holocelulosa.

Holocelulosa. Se cuantificó el contenido de holocelulosa empleando clorito de sodio de acuerdo con el procedimiento de Wise et al. (1946). En un vaso de precipitados se vertieron $32 \mathrm{ml}$ de agua destilada a $1 \mathrm{~g}$ de harina de madera libre de extraíbles, entonces se adicionaron $0,3 \mathrm{~g}$ de clorito de sodio y 2 gotas de ácido acético glacial y la muestra se llevó a un baño de agua a $75^{\circ} \mathrm{C}$. La adición de clorito de sodio y ácido acético glacial, en las cantidades ya indicadas, se repitió cíclicamente cada hora, por un periodo total de 4 horas. Después de la cloración, la solución fue filtrada, lavada con $100 \mathrm{ml}$ de agua fría, seguida de $10 \mathrm{ml}$ de acetona; el residuo fue llevado a un horno convencional a $40{ }^{\circ} \mathrm{C}$ hasta peso constante. El contenido de holocelulosa se calculó dividiendo el peso del residuo anhidro entre el peso de la harina libre de extraíbles anhidra referido porcentualmente.

Lignina. El contenido de lignina en la harina de madera libre de extraíbles se determinó de conformidad con la técnica Runkel y Wilke (1951). A $1 \mathrm{~g}$ de muestra de harina de madera libre de extraíbles se le adicionaron $50 \mathrm{ml}$ de ácido sulfúrico al $72 \%$ y 50 $\mathrm{ml}$ de ácido bromhídrico al $40 \%$, agitándola y dejándola reposar por 2 horas. Posteriormente, se le agregaron $200 \mathrm{ml}$ de agua destilada y se llevó a ebullición por 5 minutos. Finalmente, se filtró en embudos Büchner y las muestras se lavaron en repetidas ocasiones hasta eliminar los residuos de ácido. Para finalizar se llevaron a peso constante en un horno a $103^{\circ} \mathrm{C}$. El contenido de lignina se calculó dividiendo el peso de la muestra anhidra entre el peso de la harina libre de extraíbles anhidra referido porcentualmente.

Determinación de taninos. Para esta determinación se emplearon $5 \mathrm{~g}$ de harina de madera de mallas 40 y 60 . Cada muestra fue colocada en un matraz Erlenmeyer en un baño de agua con una relación sólido-líquido de 1/15 empleando agua destilada, a una temperatura de $87^{\circ} \mathrm{C}$, durante 120 minutos (Pedraza, 2006). Posteriormente se enfrió y filtró, el extracto se aforó con agua destilada en un matraz de $100 \mathrm{ml}$. El extracto total (cantidad de sólidos solubles) se determinó gravimétricamente depositando $50 \mathrm{ml}$ en un crisol de porcelana y llevado a un horno a $105^{\circ} \mathrm{C}$ hasta peso constante. Los $50 \mathrm{ml}$ restantes se tomaron para la determinación del número de Stiasny; esta solución se mezcló con $10 \mathrm{ml}$ de formaldehído concentrado en medio ácido $(5 \mathrm{ml}$ de $\mathrm{HCl}$ concentrado). La mezcla se mantuvo en ebullición bajo reflujo por 30 minutos. El precipitado obtenido se filtró con vacío en un filtro de vidrio (previamente secado y tarado) y se lavó con agua destilada. Luego, se llevó a peso constante en un horno a $103^{\circ} \mathrm{C}$. Tanto el contenido de sólidos del extracto total como el número de Stiasny se calculó gravimétricamente dividiendo el peso del residuo entre el peso inicial de la muestra y se expresó en porcentaje. El porcentaje de taninos condensados en la madera se calculó multiplicando el número de Stiasny en fracción por el rendimiento en sólidos obtenidos en cada extracto. 


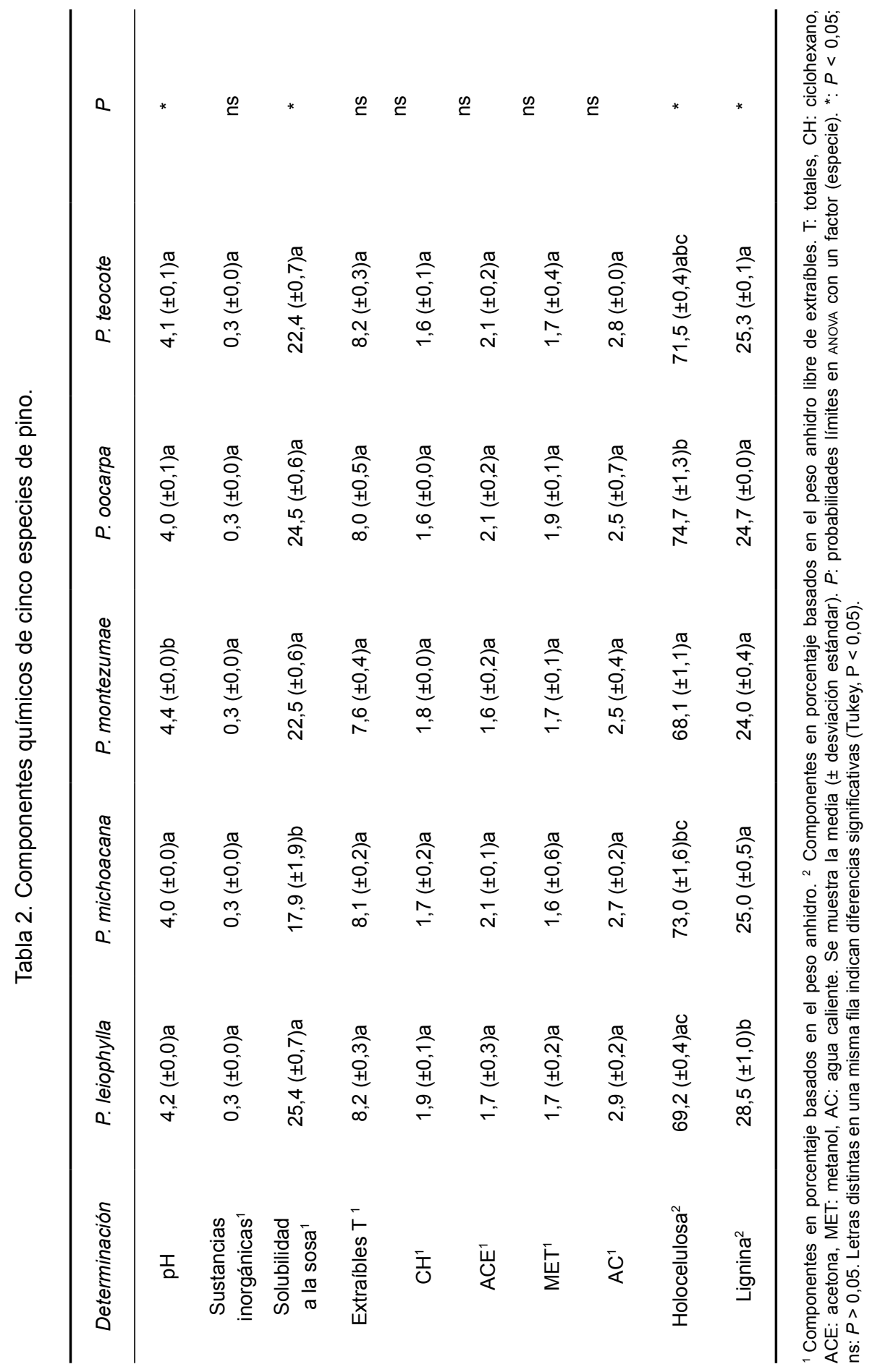


Análisis estadísticos

Para establecer la significancia de las diferencias entre las especies, los valores de los componentes químicos obtenidos fueron sometidos a análisis de varianza y a la prueba de Tukey para comparar parejas de medias de los tratamientos (especies). Para los valores de taninos se trabajó con un diseño factorial $5 \times 2$, donde los factores cualitativos a analizar fueron el tipo de material (harina de madera de la especie de pino) y el tamaño de partícula de este material. El valor de probabilidad alfa, establecido para calificar como significativas a las diferencias encontradas en las fuentes de variación, fue de 0,05. Los resultados obtenidos se procesaron mediante el software Statistica ver. 7.0.

\section{RESULTADOS}

Los resultados promedio y la desviación estándar de los componentes químicos para cada una de las especies de pino estudiadas se muestran en la tabla 2 . En esta tabla, las letras minúsculas representan la comparación entre especies (columnas), los valores con la misma letra indican que no existe diferencia significativa $(P>0,05)$. Las sustancias inorgánicas determinadas por espectroscopia de rayos $\mathrm{x}$ se muestran en la tabla 3 .

Finalmente, los resultados de los rendimientos promedio y desviación estándar del extracto total, del número de Stiasny y de los taninos condensados se presentan en la tabla 4 . En esta tabla las letras minúsculas representan la comparación entre especies (columnas) y las letras mayúsculas representan la comparación entre tamaño de partícula (malla 40 y malla 60) para cada variable (extracto total, número de Stiasny y taninos condensados). Para ambos casos, los valores con la misma letra indican que no existe diferencia significativa $(P>0,05)$, ya sea entre especies o entre tamaño de partícula. En las tablas 5, 6 y 7 se muestran los análisis de varianza para cada una de las variables de estudio.

\section{DISCUSIÓN}

$p H$. El valor de $\mathrm{pH}$ para las cinco especies estudiadas fue moderadamente ácido (Tabla 2), encontrándose diferencia significativa $(P<0,05)$ entre el $\mathrm{pH}$ de $P$. montezumae $(4,4)$ y el $\mathrm{pH}$ del resto de las maderas. Los valores $\mathrm{pH}$ obtenidos en este estudio son ligeramente más ácidos que los reportados en madera de 6 pinos mexicanos: de 4,09 a 5,63 en albura y de 4,26 a 5,23 (Rutiaga, 2001; Revilla, 2011) y aún más ácidos que en pinos europeos ( $\mathrm{pH}$ de 4,9 a 6,0) (Fengel y Wegener, 1989). Esta variación se debe a que el grado de acidez de la madera se ve afectado por la localización en el árbol de la madera analizada, la estación, los factores climáticos, la cantidad y el tipo de extraíbles, y por la presencia de grupos ácidos y ácidos libres. El pH moderadamente ácido en las maderas en estudio puede afectar su utilización: en contacto con metales puede corroer, también se puede ver afectada la fijación de sustancias preservantes, el fraguado de los adhesivos, la fabricación de tableros y productos plastificados y el proceso de pulpeo (Fengel y Wegener, 1989; Poblete et al., 1991; Poblete y Roffael, 2004).

Sustancias inorgánicas. El contenido de sustancias inorgánicas fue de $0,3 \%$ en las especies en estudio (Tabla 2), por lo que no se encontró diferencia significativa en esta determinación $(P=0,3453)$. El resultado aquí obtenido es cercano al reportado para $P$. sylvestris $(0,27 \%)$ (Balaban y Yilgör, 1995) y para $P$. pringlei $(0,34 \%)$ (Ávila, 2011), mayor al encontrado en duramen $(0,08 \%)$ y en albura $(0,16 \%)$ en $P$. pseudostrobus (Rutiaga, 2001), y menor al rango indicado por Revilla (2011) para 5 


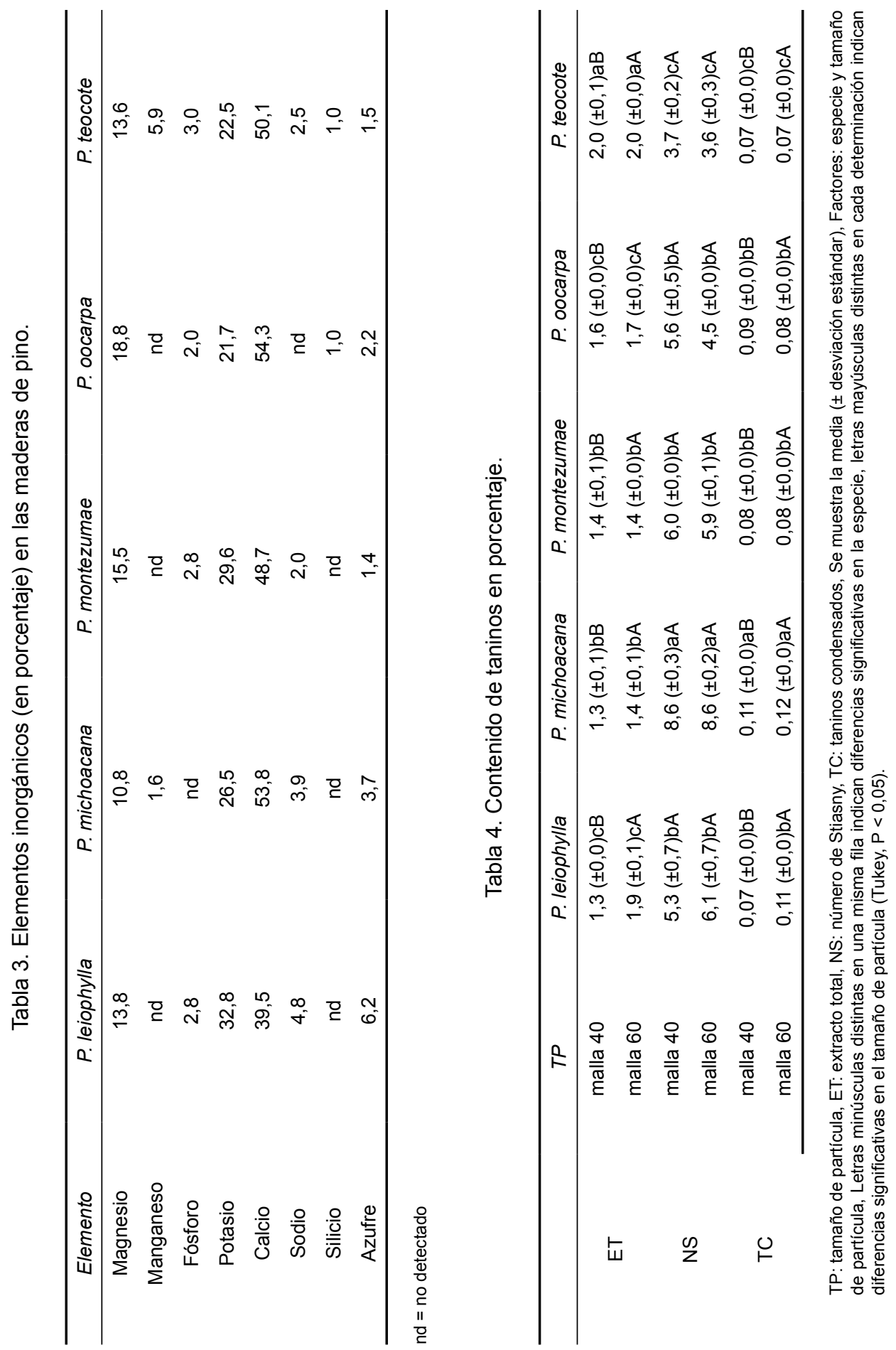



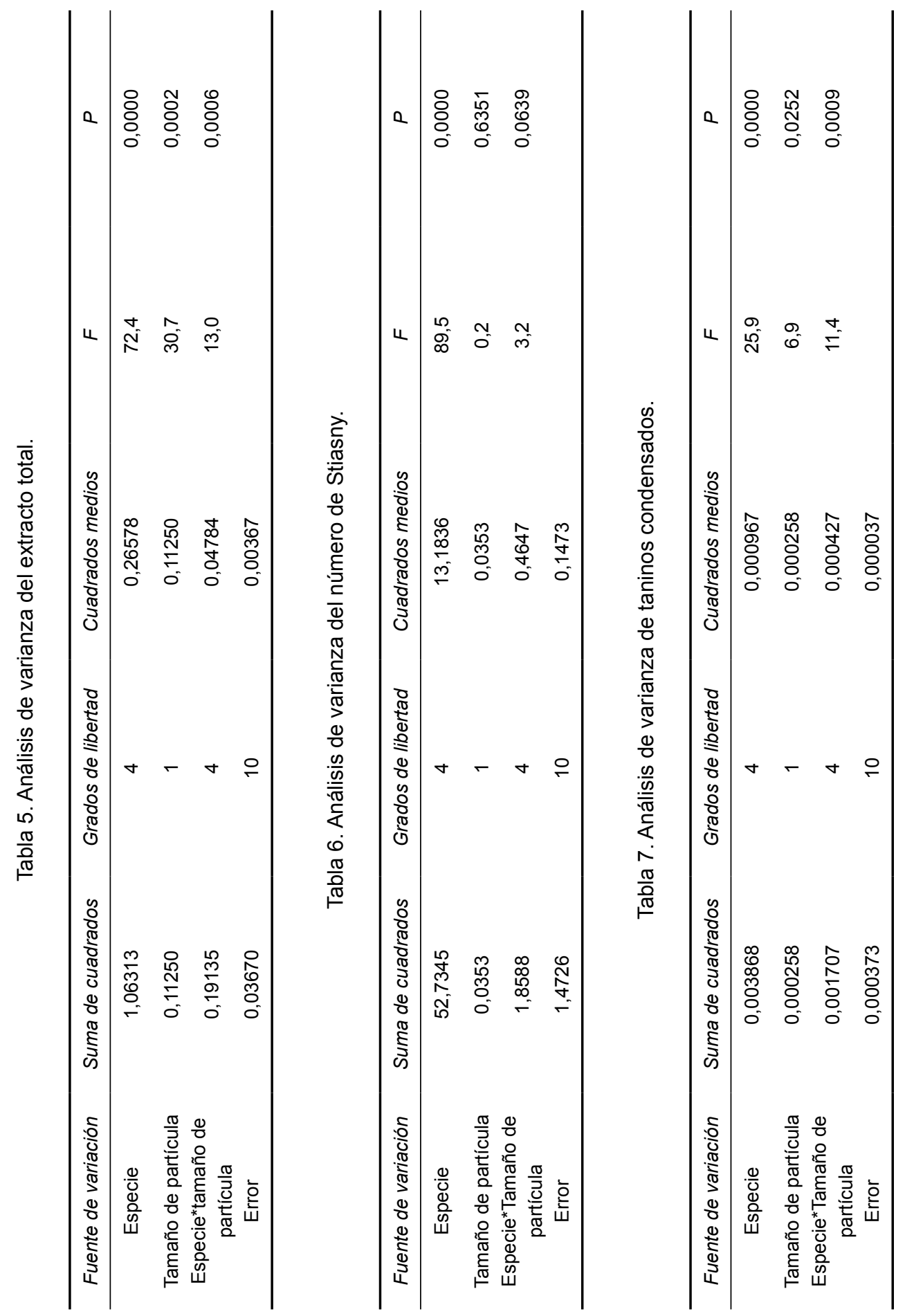
pinos mexicanos $(0,39 \% \quad 0,52 \%)$; sin embargo, los resultados se encuentran dentro del rango porcentual $(0,1 \%-0,8 \%)$ reportado para algunas especies de pino extranjeras (Fengel y Wegener, 1989; González, 2005; Rodríguez, 2005; Fonseca, 2006). En cuanto a la variabilidad de estos componentes en el árbol, González (2005) y Fonseca (2006) determinaron que no existe diferencia significativa del contenido de sustancias inorgánicas a tres alturas del fuste, cuando analizaron nueve ejemplares de P. oocarpa $(0,2 \%-0,6 \%)$ y P. maximinoi $(0,1 \%-0,7 \%)$ respectivamente. Por su parte, Rodríguez (2005) reporta un mayor contenido en la parte alta $(0,5 \%)$ que en la parte baja $(0,3 \%)$ en nueve ejemplares de P. pseudostrobus.

El resultado del microanálisis de las cenizas, mediante rayos $X$, indica que los elementos encontrados en mayor proporción son calcio, potasio y magnesio (Tabla $3)$; estos elementos son los principales componentes de las sustancias inorgánicas en la madera (Fengel y Wegener, 1989), también reportados en otros pinos mexicanos en ese orden de importancia (Rutiaga, 2001; Revilla, 2011) y mayor proporción de magnesio que potasio en P. pringlei (Ávila, 2011). En este estudio, el silicio sólo se encontró en $P$. oocarpa $(1,0 \%)$ y $P$. teocote $(1,0 \%)$; algunos autores consideran que una concentración de entre 1,0\% y 3,0\% representa problemas para la transformación de la madera, principalmente en el desafilado de las herramientas de corte (Honorato y Hernández, 1998), siendo la rapidez de desafilado en la sierra banda en función del contenido de silicio en la madera (Kirlov, 1980). No obstante, la cantidad de este elemento químico detectada en las maderas señaladas puede considerarse de bajo impacto en el desafilado de herramientas de corte al procesar este material.

Solubilidad a la sosa. Los resultados obtenidos muestran que $P$. michoacana tiene baja solubilidad en sosa $(17,9 \%)$ y resultó ser estadísticamente significativa $(P<0,05)$ con respecto a las otras cuatro especies estudiadas (Tabla 2). Los extraíbles solubles en sosa pueden ser carbohidratos de bajo peso molecular, principalmente hemicelulosas, los que están relacionados con el grado de pudrición por hongos o el deterioro por calor, luz u oxidación (TAPPI T 212 om-98), esto podría indicar que la madera de $P$. michoacana es más resistente al deterioro en comparación con las demás maderas. Los valores obtenidos para todas las especies en estudio se encuentran por arriba del contenido reportado en la literatura para diferentes especies de pino, de 11\% - 16\% (Rowell, 2005; Ávila, 2011).

Sustancias extraíbles. De acuerdo con los resultados obtenidos (Tabla 2 ) no se presentó diferencia significativa $(P=0,5537)$ para los extractos totales de las especies, ni para los diferentes solventes (ciclohexano $P=0,1355$, acetona $P=0,2057$, metanol $P=0,9999$, agua caliente $P=0,9999)$. La mayor solubilidad de la madera se logró con agua caliente. Los rendimientos alcanzados son ligeramente superiores al rango encontrado por otros autores para especies de pino $(2,4 \%-7,7 \%)$ (Fengel y Wegener, 1989; Rodríguez, 2005; Fonseca, 2006), e inferiores a los calculados en P. oocarpa $(10,89 \%)$ (González, 2005), duramen de P. pseudostrobus $(13,2 \%)$ (Rutiaga, 2001) y albura y duramen de $P$. cembroides, $P$. maximartinezii, $P$. johannisy P. pinceana $(11,5 \%-33,7 \%)$ (Revilla, 2011). La relación entre el contenido de extraíbles y las propiedades físicas y tecnológicas dependen del contenido de extraíbles, de su tipo y de su localización; por un lado, en algunas especies pueden aumentar la estabilidad dimensional y la resistencia mecánica; por el otro, pueden disminuir el punto de saturación de la fibra y el contenido de humedad en equilibro y afectar otras propiedades tecnológicas (Poblete et al., 1991; Ávila y Herrera, 2012). 
Holocelulosa. Se encontró diferencia significativa $(P<0,05)$ en el contenido de holocelulosa en las especies estudiadas: $P$. montezumae presentó un valor relativamente bajo $(68,1 \%)$, pero dentro del rango de 63,6 al $68,7 \%$ determinado en pinos mexicanos (Rutiaga, 2001; Ávila, 2011; Revilla, 2011). Para el resto de los pinos en estudio, el contenido de carbohidratos fue relativamente alto $(69,2 \%-74,7 \%)$ (Tabla 2). Los valores determinados se encuentran en el rango reportado en la literatura para maderas de coníferas $(64,0$ - 82,5\%) (Fengel y Wegener, 1989; Rowell, 2005).

Lignina. El valor más alto de lignina Runkel lo presentó $P$. leiophylla $(28,5 \%$ ) con diferencia estadísticamente significativa $(P<0,05)$ respecto al resto de las especies estudiadas (Tabla 2); este valor es cercano al publicado para duramen de $P$. pseudostrobus $(27,6 \%)$, pero ligeramente mayor al encontrado en albura de esta misma especie (26,6\%) (Rutiaga, 2001). Los contenidos de lignina de las especies en estudio se encuentran dentro del intervalo de lignina Klason publicado para maderas de pino mexicanas $(23,6 \%$ - 31.2\%) (Ávila, 2011; Revilla 2011) y para maderas de pino extranjeras (22,0\% 35,0\%) (González, 2005; Rodríguez, 2005; Rowell, 2005; Fonseca, 2006). En este estudio se encontró que el contenido de lignina Runkel en $P$. oocarpa fue menor $(24,7 \%)$ al determinado para esta misma especie por González (2005), pero por el método Klason $(28,1 \%-30,4 \%)$, lo que pudiera explicar la variación encontrada. González (2005) y Fonseca (2006) determinaron que no hay diferencia significativa para los contenidos de lignina a tres alturas del árbol en $P$. oocarpa y $P$. maximinoi, contrario a lo encontrado en $P$. pseudostrobus (Rodríguez, 2005). Se conoce que el contenido de lignina desempeña un papel importante en el comportamiento de la madera ante los cambios dimensionales por variaciones en el con- tenido de humedad (Bárcenas y Dávalos, 1999); así, la baja contracción máxima (radial 1,6\%, tangencial 2,0\%) encontrada para la madera de $P$. leiophylla (Herrera y Bocanegra, 1996) pudiera explicarse por su alto contenido de lignina (Tabla 2), comparada con la contracción, relativamente alta publicada para las maderas de $P$. montezumae (radial $5,0 \%$, tangencial $6,6 \%$ ), de $P$. oocarpa (radial 3,3\%, tangencial 4,4\%) (Herrera y Bocanegra, 1996) y para $P$. michoacana (radial $3,1 \%$, tangencial 6,9\%) (Sotomayor et al., 2010), en cuyo caso la concentración de lignina aquí determinada en estas maderas es menor al encontrado en $P$. leiophylla (Tabla 2).

Taninos. Debido a la mayor penetración del solvente en partículas más pequeñas, el contenido de extractos totales muestra mayores rendimientos con la partícula retenida en la malla $60(P<0,05)$ y respecto a la especie se encontró mayor contenido en $P$. teocote $(P<0,05)$ (Tabla 4$)$; asimismo, la interacción entre los dos factores: especie y tamaño de partícula también fue significativo $(P<0,05)$ (Tabla 5$)$. El número de Stiasny fue mayor en la madera de $P$. michoacana $(8,6 \%)$ y menor para $P$. teocote $(3,6 \%)$ considerando que esta última especie presentó el mayor rendimiento de extractos totales. Los valores fueron significativos para el factor especie $(P<0,001)$, no así para el factor tamaño de partícula $(P=0,6351)$ ni para la interacción entre los factores especie y tamaño de partícula $(P=0,0639)$ (Tabla 6). La determinación del número de Stiasny es importante al indicar qué porcentaje de los extraíbles obtenidos tienen la capacidad de reaccionar con el formaldehído, lo que indirectamente se relaciona con la posibilidad de obtener taninos para uso industrial (Poblete y Roffael, 2004). En cuanto al contenido de taninos condensados, se presentó diferencia significativa en el factor especie $(P<0,05)$, en el tamaño de partícula $(P<0,05)$ y en la interacción de los factores especie y tamaño 
de partícula $(P<0,05)$ (Tabla 7$)$. Debido a que, en general, las cortezas contienen mayor contenido de polifenoles que la madera, en este estudio se obtuvieron valores menores que los encontrados por Rosales y González (2003) para los extractos acuosos de las cortezas de $P$. leiophylla (80,0\% número de Stiasny y $5,88 \%$ de taninos condesados) y $P$. teocote $(57,4 \%$ y $3,02 \%$ respectivamente).

\section{CONCLUSIONES}

La composición química básica, determinada en la mezcla de madera de albura y duramen, para las cinco especies de pino, coincide en general con datos de la literatura para maderas de pino mexicanas y extranjeras, aun cuando este estudio solamente se realizó con un árbol por especie. Los contenidos de los principales componentes químicos variaron de 4,0 a 4,4 para el $\mathrm{pH} ; 0,3 \%$ de cenizas en todas las especies, con mayor presencia de calcio, potasio y magnesio; de $17,9 \%$ a $25,4 \%$ de solubilidad a la sosa; de $7,6 \%$ a $8,2 \%$ de extraíbles totales, en la extracción secuencial la mayor solubilidad se presentó con agua caliente, y en proporciones similares fue para ciclohexano, acetona y metanol; de $68,1 \%$ a $74,7 \%$ de holocelulosa, de $24,0 \%$ a $28,5 \%$ de lignina. El valor $\mathrm{pH}$, la solubilidad en sosa, la holocelulosa y la lignina presentaron una variación estadística significativa $(P<0,05)$ entre especies.

Para el porcentaje de extracto total y de taninos condensados se encontró efecto significativo entre especies, tamaño de partícula y en la interacción entre especie - tamaño de partícula, mientras que para el número de Stiasny sólo hubo diferencia significativa para la especie. La madera de $P$. michoacana presentó el número de Stiasny y el porcentaje de taninos condensados más alto, por el contrario, la madera de $P$. teocote presentó los porcentajes más bajos, incluso cuando esta especie arrojó el extracto total mayor.

\section{RECONOCIMIENTOS}

Se agradece a la Coordinación de la Investigación Científica de la Universidad Michoacana el apoyo al Proyecto CICJGRQ-21.3, dentro de la cual se desarrolló este estudio. Al maestro en ciencias Xavier Madrigal Sánchez y a la bióloga Lydia I. Guridi Gómez la donación del material de estudio del proyecto "Los árboles del municipio de Morelia, Michoacán, México" Conacyt-UMSNH y la información de los datos de colecta.

\section{REFERENCIAS}

Ambriz Parra, J.E., L.I. Guridi-Gómez, G.I. Martínez-Sánchez y T. Ochoa-Álvarez. 2002. Anatomía de la madera e índices de calidad de pulpa de dos variedades de pino lacio (Pinus michoacana var. michoacana Martínez y Pinus michoacana var. cornuta Martínez) de un bosque de pinoencino del municipio de Morelia, Michoacán, México. Ciencia y Tecnología de la Madera 7:2-16.

Aquino-González, L.V., J. RodríguezRamírez, L.L. Méndez-Lagunas y S. Sandoval-Torres. 2010. Evaluación de programas de secado para madera de chalamite (Pinus pseudostrobus). Madera y Bosques 16(2):35-46.

Ávila Calderón, L.E.A. 2011. Evaluación de los componentes químicos básicos de la madera de Pinus pringlei infectada por el muérdago Psittacanthus macrantherus. Ciencia Nicolaita 54:7-16.

Ávila, L.E. y M.A. Herrera. 2012. Efecto de los extraíbles en tres propiedades 
físicas de la madera de Enterolobium cyclocarpum procedente de Michoacán, México. Bosque 33(2):115-120.

Balaban, M. y N. Yilgör. 1995. The acidity of heartwoods and softwoods grown in Turkey. Holz als Roh- und Werkstoff. 53:332.

Bárcenas-Pazos, G. y R. Dávalos-Sotelo. 1999. Efecto de la lignina en las contracciones de la madera, revisión bibliográfica. Madera y Bosques 5(1):13-26.

Cruz de León, J. y J.E. Ambriz-Parra. 2004. Impregnación de madera en autoclave con sales de cobre, cromo y arsénico por el método Bethell. Ciencia Nicolaita 37:177-188.

Fengel, D. y G. Wegener. 1989. Wood: chemistry, ultrastructure, reactions. Walter de Gruyter. Berlín. 613 p.

Fonseca M., M.R. 2006. Determinación de la composición química de la madera de pino candelillo (Pinus maximinoi H. E. Moore) procedente de la finca Río Frío, Tactic, Alta Verapaz. Tesis profesional. Escuela de Ingeniería Química. Universidad de San Carlos de Guatemala. Guatemala. 125 p.

González Pimentel, M.R. 2005. Determinación de la composición química de la madera del pino ocote (Pinus oocarpa Schiede ex Schltdl) procedente de plantación en Cucanjá, Tucurú, Alta Verapaz. Tesis profesional. Escuela de Ingeniería Química. Universidad de San Carlos de Guatemala. Guatemala. 110 p.

Herrera Ferreyra, M.A. y S. BocanegraOjeda. 1996. Características físicomecánicas de la madera de 15 especies del municipio de Morelia.
Ciencia y Tecnología de la Madera 10:3-11.

Honorato Salazar, A. y J. Hernández Pérez. 1998. Determinación de componentes químicos de la madera de cinco especies de encino del estado de Puebla. Madera y Bosques 4(2):79-93.

Kirlov, A. 1980. Toward the re-appraisal of the influence of basic wood characteristics on sawblade potential. Holz als Roh- und Werkstoff. 38:145-149.

Madrigal Sánchez, X. y L.I. Guridi-Gómez. 2002. Los árboles silvestres del municipio de Morelia, Michoacán, México. Ciencia Nicolaita. 33:29-58.

Ochoa Álvarez, M.T. 2003. Características anatómicas mesurables e índices de calidad de pulpa de la madera de 11 especies de pino del municipio de Morelia, Michoacán. Tesis profesional. Facultad de Ingeniería en Tecnología de la Madera. Universidad Michoacana de San Nicolás de Hidalgo. Morelia, Michoacán, México. 54p.

Olvera-Coronel, P. 1981. Anatomía de la madera de 7 especies del género Pinus. Boletín técnico núm. 71. Instituto Nacional de Investigaciones Forestales, México. 11p.

Olvera-Coronel, P. 1985. Características anatómicas de 7 especies del género Pinus. Boletín técnico núm. 126. Instituto Nacional de Investigaciones Forestales, México. 73p.

Ortega-Escalona, E. 2001. Densidad básica y longitud de traqueidas en la madera de cinco familias de Pinus patula Schltdl. \& Cham. en dos sitios del estado de Veracruz, México. Ciencia y Tecnología de la Madera 2(5):21-41. 
Pedraza Bucio, F.E. 2006. Composición química de la corteza de cuatro especies de eucalipto y evaluación de las propiedades de sus extractos tánicos. Tesis de Maestría. Facultad de Ingeniería en Tecnología de la Madera. Universidad Michoacán de San Nicolás de Hidalgo. Morelia, Michoacán, México. 136p.

Poblete, H., S. Rodríguez y M. Zárate. 1991. Extraíbles de la madera, sus características y efectos sobre la utilización de esta materia prima. Publicación docente núm. 34. Facultad de Ciencias Forestales. Universidad Austral de Chile. Valdivia, Chile. 51 p.

Poblete, H. y E. Roffael. 2004. Acidez de la corteza de algunas especies nativas chilenas. Bosque 25(3):73-78.

Revilla González, E. 2011. Química de la madera de cuatro pinos mexicanos de la subsección cembroides. Tesis profesional. Programa académico de Ingeniero Forestal Industrial. Universidad Autónoma Chapingo. México. $92 \mathrm{p}$.

Rodríguez, L. M. E. 2005. Determinación de los componentes químicos de la madera de pino blanco (Pinus pseudostrobus Lindl.) proveniente de la finca Las Victorias, Patzún, Chimaltenango. Tesis profesional. Escuela de Ingeniería Química. Universidad de San Carlos de Guatemala. Guatemala. $90 \mathrm{p}$.

Rojas G., F. y L. Villers R. 2005. Comparación de dos métodos para estimar la densidad de la madera de Pinus hartwegii Lindl. del Volcán La Malinche. Madera y Bosques 11(1):63-71.

Rosales-Castro, M. y R.F. GonzálezLaredo. 2003. Comparación del contenido de compuestos fenólicos en la corteza de ocho especies de pino. Madera y Bosques 9(2):41-49.

Rowell, R. 2005. Handbook of wood chemistry and wood composites. Taylor \& Francis. United States of America. $473 \mathrm{p}$.

Runkel, Roh y K.D. Wilke. 1951. Zur Kenntnis des thermoplastischen Verhaltens von Holz. Holz Roh Werkstoff. 9:260-270.

Rutiaga Quiñones, J.G. 2001. Chemische und biologische Untersuchungen zum Verhalten dauerhafter Holzarten und iher Extrakte gegenüber holzabbuenden Pilzen. Buchverlag Gräfelfing. Müchen. 201 S.

Sánchez-González, A. 2008. Una visión actual de la diversidad y distribución de los pinos de México. Madera y Bosques 14(1):107-120.

Sandermann, W. y M. Rothkamm. 1959 Über die Bedeutung der $\mathrm{pH}$-Werte von Handelshölzern und deren Bedeutung fur die Praxis. Holz als Roh- und Werkstoff. 17:433-440.

Semarnat (Secretaría de Medio Ambiente y Recursos Naturales). 2012. Anuario estadístico de la producción forestal 2011. [Internet]. Disponible en: http://www.semarnat.gob.mx/temas/ gestionambiental/forestalsuelos/ Anuarios/ANUARIO_2011. Fecha de acceso: 17/02/2013.

Silva-Arredondo, F.M. y J.J. Návar-Cháidez. Estimación de la densidad de madera en árboles de comunidades forestales templadas del norte del estado de Durango, México. Madera y Bosques 18(1):77-88.

Sotomayor-Castellanos, J.R., L.J. GarcíaMariscal, C.E. Moya-Lara y J.B. 
Olguín-Cerón. 2010. Higroscopía y anisotropía de la madera de Pinus michoacana, Pinus douglasiana y Pinus pringlei. Investigación e Ingeniería de la Madera 6(3):3-32.

Technical Association for the Pulp and Paper Industries (TAPPI). 2000a. Ash in wood, pulp, paper and paperboard: combustion at $525^{\circ} \mathrm{C}$. TAPPI Test method T 211 om-93. TAPPI Press. Atlanta, Georgia. EUA. 4 p.
Technical Association for the Pulp and Paper Industries (TAPPI). 2000b. One percent sodium hydroxide solubility of wood and pulp. TAPPI Test method T 212 om-98. TAPPI Press. Atlanta, Georgia. EUA. 4 p.

Wise L.E., M. Murphy y A.A. D'Addieco. 1946. Chlorite holocellulose, its fractionation and bearing on summative wood analysis and on studies on the hemicelluloses. Pap. Trade J. 122 (3):35-43. cinco especies de pino del municipio de Morelia, Michoacán. Madera y Bosques 19(2):21-35. 